\title{
Application of Magnetohydrodynamic propulsion technology in demonstration physics experiment
}

\author{
Guang HU *, Qingsong JIANG, Jun ZHANG, Yalu TANG
}

Faculty of Mathematics and Physics, Huaiyin Institute of Technology, Huai'an 223003, China

Corresponding: *E-mail: hygxyhg@126.com

Keywords: Magnetohydrodynamic; Propulsion; Physical experiment

\begin{abstract}
The propulsion technology of magnetohydrodynamics is considered as the interdiscipline, involving the electromagnetism, fluid mechanics, electrochemistry. The magnetohydrodynamic propulsion device is applied to boats. The boats show the high speed in the quiet mode, and have advantages of concealment, simple operation, and high safety performance. Thus, the magnetohydrodynamic propulsion device can be used as the main power system of boats in future. In this paper, the demonstration experiment instrument for magnetohydrodynamic propulsion is designed and constructed by using boat as mold. The Solidworks software is used to design the boat mold. The BA5104/SM5032C integrate circuit of the eight road Infrared remote controller is applied as the control circuit. The propeller can be controlled by the codec. The rechargeable batteries are used as the propulsion power of magnetohydrodynamics. The push-pull circuit is used as the drive circuit. The propulsion technology with a two channels is used to generate power. In our experiment, $\mathrm{NaCl}$ is added into purified water to simulate seawater. The research results will provide a chance to explore magnetofluid technology and application in practice.
\end{abstract}

\section{Introduction}

The interaction force between the current in the sea water and the magnetic field is used as the propulsion force in the propulsion technology of magnetohydrodynamics [1, 2]. This mean that the reverse thrust is generated by the movement of the sea water. The kinetic energy of the fluid has been obtained by converting electrical energy. Therefore, the traditional propeller can be replaced by the propulsion technology of magnetohydrodynamics. The propulsion technology of magnetohydrodynamics is considered as the interdiscipline, involving the electromagnetism, fluid mechanics, electrochemistry, and a comprehensive, sophisticated science and technology.

The propulsion device is used in magnetohydrodynamic propulsion without mechanical movement, such as propeller blades, gear transmission and shaft pump. Some technical issues, such as mechanical rotation, noise and power constraints, can be resolved by using he magnetohydrodynamic propulsion [3,4]. Therefore, the boats with the magnetohydrodynamic propulsion can sail with high speed in a quiet state. There are many advantages, for example, concealment, simple operation, and high safety performance. We can suppose that the magnetohydrodynamic propulsion technology can be used as the main driving force system of the boats in the future.

\section{Design ideas of the magnetohydrodynamic propulsion device}

The demonstration experimental instrument of magnetohydrodynamic propulsion consists of the boat mold, propulsion device, power and control, and seawater simulation. The Solidworks software is used to design the boat mold. The propulsion technology with a two channels is used to control the forward, turning, and back of the boat by changing the electrode method. The rechargeable batteries are used as the propulsion power of magnetohydrodynamics. The remote control method is used to control the experimental instrument. $\mathrm{NaCl}$ is added into purified water to simulate seawater with blue pigment. Figure 1 is the design boat mold for the demonstration experiment instrument of magnetohydrodynamic propulsion. 


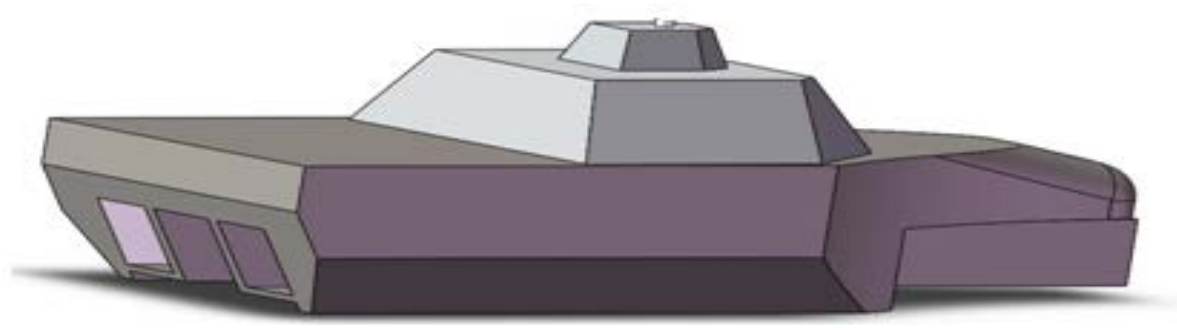

Figure 1. The boat mold for the magnetohydrodynamic propulsion.

\section{Working principle of the magnetohydrodynamic propulsion device}

The two channels have been used in the magnetohydrodynamic propulsion device. A rectangle cavity composes of a permanent magnet and two pieces of inert electrodes for each channel [5]. Figure 2 shows the work principle of the magnetohydrodynamic propulsion device. The uniform magnetic field is vertically downwards from the high strength permanent magnet. The electrode 1 is obtained a positive voltage (high voltage). The electrode 2 is obtained a negative voltage (low voltage). The electric field is formed between the electrode 1 and the electrode 2 . The conductive circuit can be formed because the internal channel is filled with sea water. The current direction is the same with the direction of electric field. The Ampere force for sea water can be described as $\mathrm{d} \overrightarrow{\mathrm{F}}=I \mathrm{~d} \overrightarrow{\mathrm{b}} \times \vec{B}$, which results in the movement of the boat, as shown in Figure 2. The voltaic polarity can be changed by controlling the electrode 1 and the electrode 2 . The movement direction of boat can be also changed by controlling the direction of the ampere force.

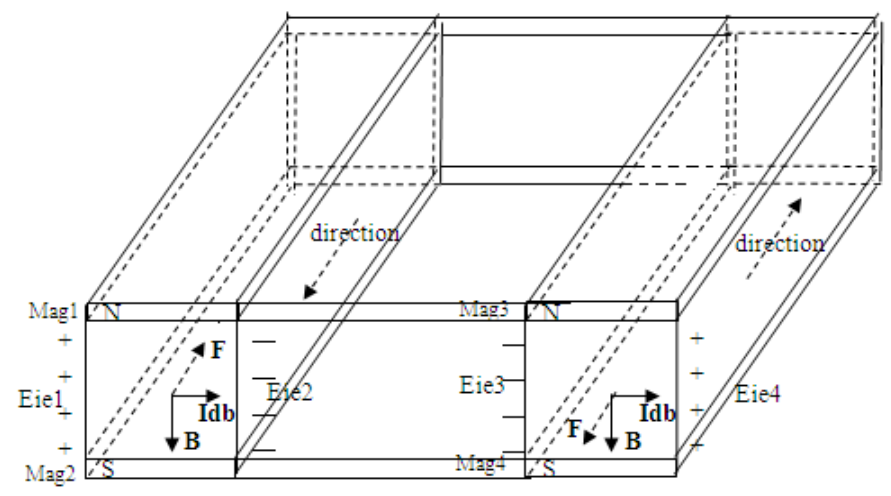

Figure 2. Principle and structure of magnetohydrodynamic propulsion device

Table 1: The relationship between the polarity of the electrode and the operation direction of the ship

\begin{tabular}{|c|c|c|c|c|}
\hline Action & Electrode 1 & Electrode 2 & Electrode 3 & Electrode 4 \\
\hline Forward & + & - & + & - \\
\hline Back & - & + & - & + \\
\hline Turn left & + & - & - & + \\
\hline Turn right & - & + & + & - \\
\hline
\end{tabular}

The thrust is not only strongly dependent on the magnetic flux density B and the channel width b, but also dependent on the current I between the two electrodes. The current I can be defined by the ratio of the voltage $\mathrm{U}$ between the two electrodes and the resistance $\mathrm{R}$ of the simulated sea water. 


\section{The principle of the control circuit}

Figure 3 shows the circuit principle of the magnetohydrodynamic propulsion demonstration instrument. The control circuit of the magnetohydrodynamic propulsion demonstration instrument composed of a remote control transmitting circuit, remote control receiving circuit, charging circuit and a driving circuit of a rechargeable battery.

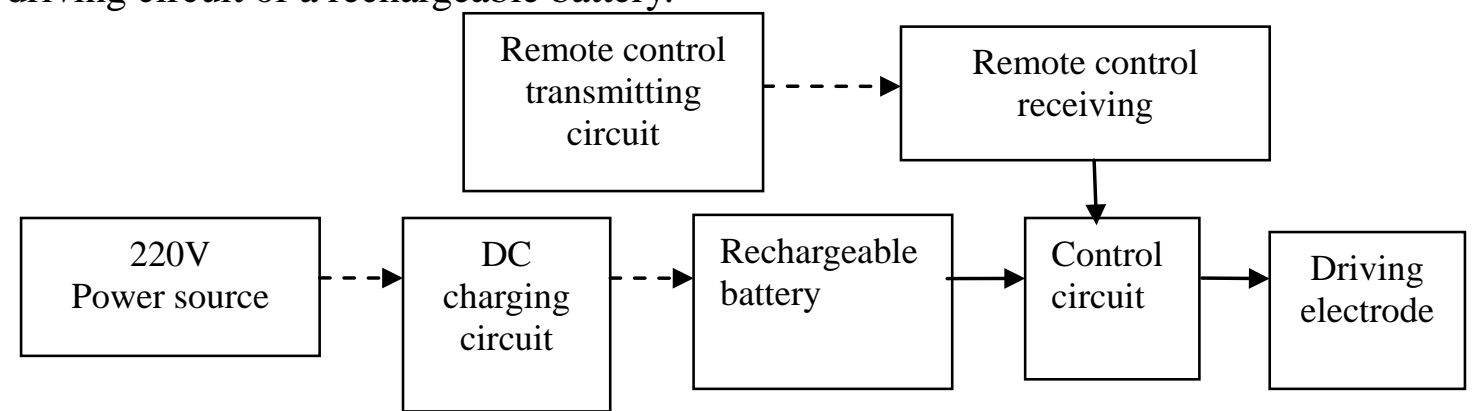

Figure 3. The circuit principle

Figure 4 and figure 5 shows the circuit principle of the BA5104/SM5032C , BA5104/SM5032C integrate circuit. The BA5104/SM5032C integrate circuit of the eight road wireless remote controller is applied as the control and receiving circuit. As we all know that the BA5104/SM5032C integrates circuit is considered as remote transmitting and receiving integrated circuit with multi functions. The BA5104/SM5032C integrates circuit has many advantages [6], such as the signal modulation, demodulation, error identification, less circuit components, good anti-interference, low power consumption, and flexible and convenient for application in our experiment.The(12) and (13)feet linked with internal clock circuit of BA5104, which connected with an external $455 \mathrm{KHZ}$ crystals oscillators and C1 and C2, the oscillation circuit Produce $38 \mathrm{KHZ}$ carrier frequency. IC encode the data from $\mathrm{K} 1-\mathrm{K} 8$ and $\mathrm{C} 1, \mathrm{C} 2$, and then serial output infrared pulse modulated carrier signal through (15) foot. (14) foot shows the state of launch. (1) and (2) foot is encode input side, we can set four different encoding. Fig. 4 shows the infrared remote-control circuit.

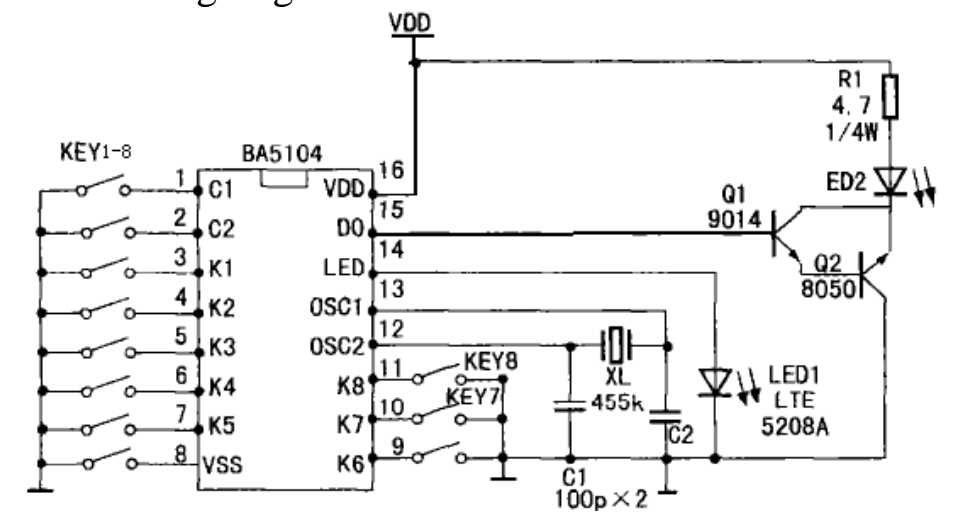

Figure4: The integrate circuit of the eight road infrared remote controller

The selected infrared signal is picked up and pre-amplifie by BA5302 infrared sensor, then the selected signal was demodulated and was send to (2) foot DI side of SM5302C. After decoding by IC, the control signals was send by (3)-(10) feet. The infrared receiving circuit is shown in fig. 5. 


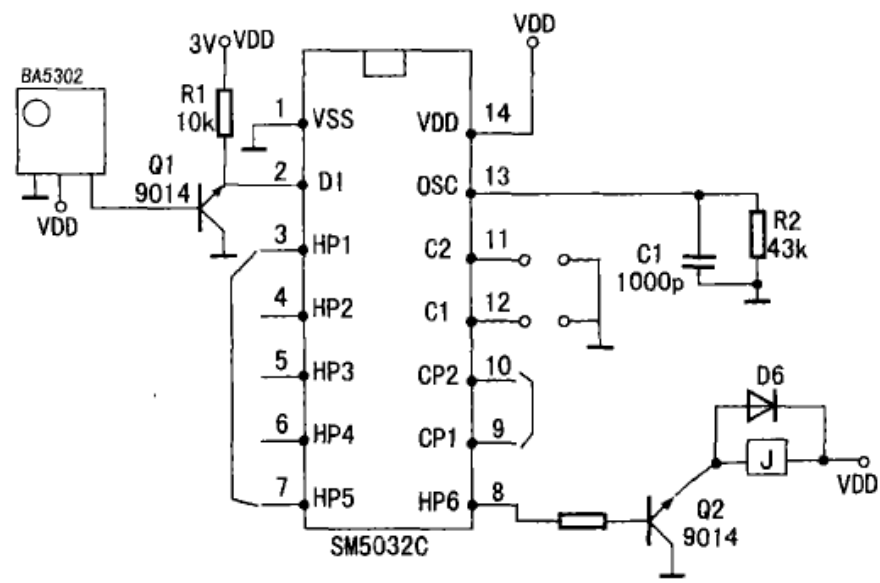

Figure 5: The receiving circuit of the eight road infrared remote controller

Figure 6 shows the control and drive circuits. The push-pull circuit with four tubes is used as the drive circuit. The encoding signal of HP1-HP6 from the receiving circuit SM5032C is connected to the power resource and in $1 \sim 4$ of the control and drive circuits. The electrodes $1,2,3$, and 4 can be controlled by the control signal. Therefore, the magnetohydrodynamic propulsion device has obtained the driving force by the control and drive circuits.

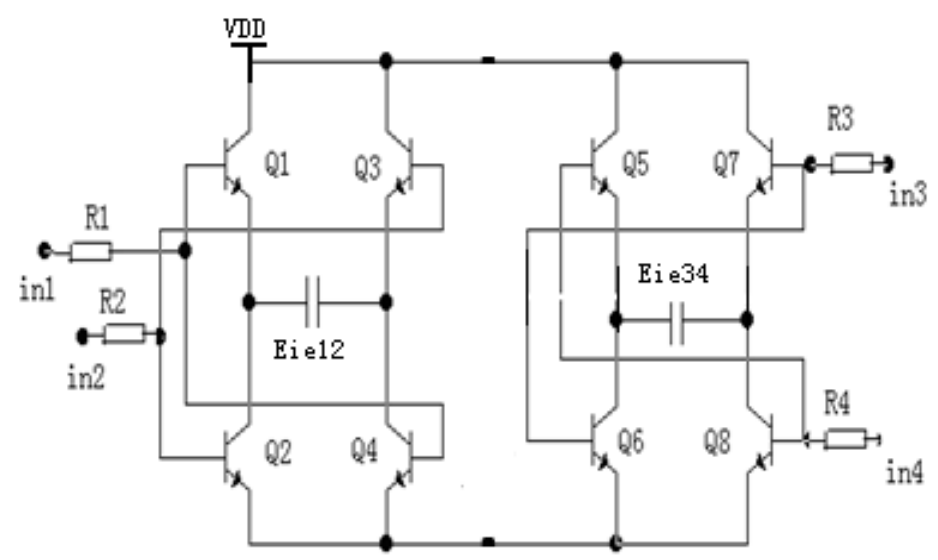

Figure 6: The control and drive circuits

Figure 7 shows the rechargeable battery charging circuit. The automatic stop charging mode is applied as the charging circuit. The charging voltage is controlled by the voltage regulator tube D1. The relay $\mathrm{J} 1$ would be opened by achieving the high voltage. Then, the charging circuit can be obtained protection by switching off.

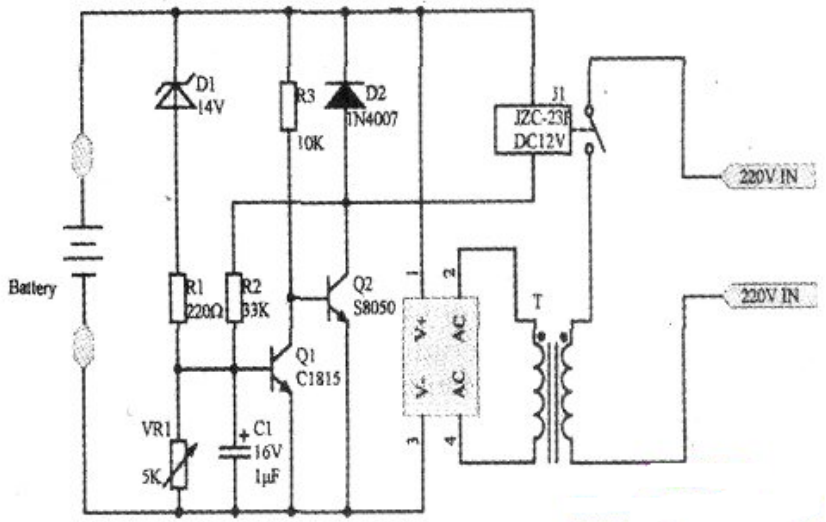

Figure 7: The rechargeable battery charging circuit

\section{Seawater simulation technologies}

In order to obtain the similar resistance, the salt $(\mathrm{NaCl})$ is added into the pure water to simulate seawater. The resistivity and concentration of the seawater are $2.5 \Omega \mathrm{m}$ and $3.8 \%$, respectively. Table 
2 shows the relationship between salt concentration and electrical resistivity at $21^{\circ} \mathrm{C}$. The solubility of $\mathrm{NaCl}$ is $36 \mathrm{~g}$ at room temperature $20^{\circ} \mathrm{C}$. Therefore, the density and concentration of the saturated $\mathrm{NaCl}$ solution are $1.12 \mathrm{~g} / \mathrm{cm}^{-3}$ and $26.47 \%$, respectively.

Table 2: Relationship between salt concentration and electrical resistivity at $21^{\circ} \mathrm{C}$

\begin{tabular}{|c|c|c|c|c|c|c|c|}
\hline Concentration & 0 & $1 \%$ & $2 \%$ & $5 \%$ & $10 \%$ & $15 \%$ & $20 \%$ \\
\hline Resistivity & 67.1 & 6.5 & 5.6 & 4.7 & 1.7 & 0.7 & 0.5 \\
\hline
\end{tabular}

\section{Parameter design and calculation of the magnetohydrodynamic propulsion device}

Table 3 shows the related parameters of the magnetohydrodynamic propulsion device. First, the length, width and height of the magnetohydrodynamic propulsion channel are needed to design. Secondly, the intensity of magnetic field and the parameters of rechargeable batteries are needed to choose. At last, the thrust is also needed to calculate.

Table 3: The related parameters of the magnetohydrodynamic propulsion device.

\begin{tabular}{|c|c|c|c|}
\hline Name & formula & value range & design value \\
\hline Channel length & $\mathrm{L}$ & $100-200 \mathrm{~mm}$ & $150 \mathrm{~mm}$ \\
\hline Channel width & $\mathrm{b}$ & $15-30 \mathrm{~mm}$ & $20 \mathrm{~mm}$ \\
\hline Channel height & $\mathrm{C}$ & $15-30 \mathrm{~mm}$ & $20 \mathrm{~mm}$ \\
\hline Salt concentration & $\eta$ & $0-26.5 \%$ & $3.8 \%$ \\
\hline Salt resistivity & $\rho$ & $60-0.6 \Omega \mathrm{m}$ & $2.5 \Omega \mathrm{m}$ \\
\hline Battery voltage & $\mathrm{U}$ & $6-30 \mathrm{~V}$ & $12 \mathrm{~V}$ \\
\hline Magnetic field & $\mathrm{B}$ & $0.3-0.8 \mathrm{~T}$ & $0.5 \mathrm{~T}$ \\
\hline Ampere force & $\mathrm{F}=\mathrm{IdlB}=\mathrm{IbB}$ & $0.048 \mathrm{~N}$ & $0.5 \mathrm{~N}$ \\
\hline Power & $\mathrm{P}=\mathrm{UI}$ & $10 \mathrm{~W}$ & \\
\hline Boat weight & $\mathrm{m}$ & $400 \mathrm{~g}$ & \\
\hline Boat speed & $\mathrm{V}$ & $0.02 \mathrm{~m} / \mathrm{s}$ & \\
\hline
\end{tabular}

\section{Operation instruction and notes of the magnetohydrodynamic propulsion device}

The detail process is given as follows.

First, according to the ratio of water and salt (100:4), a certain amount of salt is added into pure water. The simulated seawater is formed by adding an amount of blue pigment.

Second, the magnetohydrodynamic propulsion device is putted on the surface of seawater, and open power switch.

Third, the device can be kept moving status by pressing the start button (forward, stop). In the status, the device can be pressed by turn left button, turn right button and the back button. The device can stop by pressing the button of start (forward, stop) button.

Fourth, after demonstration, the device should be removed from the simulated.

Fifth, notes: the device should not be placed together with the iron, cobalt, nickel and their compounds, due to the permanent magnet in the device. The device should be protected by slightly operating.

\section{Conclusions}

In summary, the magnetohydrodynamic propulsion device has been designed. The magnetic field can be obtained by the permanent magnet. The rechargeable batteries are used as the propulsion power. The Solidworks software is used to design the boat mold. $\mathrm{NaCl}$ is added into purified water to simulate seawater. The results demonstrate that magnetofluid technology can be considered as the potential application in the modern military field. 


\section{Reference:}

[1] Li Yaxu, Liu Xiaolin Ship Magnetohydrodynamic Thruster and High Temperature Superconduction, Marine Electric \& Electronic Engineering, 2009(08). 1-4.

[2] CHEN Li-mei, CHENG Min-xi, XIAO Xiao-fang, HUANG Zuo-hua, Measurement of the Relationship between Conductivity of Salt Solution and Concentration and Temperature, Research and exploration in laboratory, 2010(05). 39-41.

[3] Mohsen Sheikholeslami, Davood Domiri Ganji, Ferrohydrodynamic and magnetohydrodynamic effects on ferrofluid flow and convective heat transfer, Energy,75 (2014) 400-410.

[4] C. Paz-Soldan, W. F. Bergerson, M. I. Brookhart, D. A. Hannum, R. Kendrick, G. Fiksel, C. B. Forest, The rotating wall machine: A device to study ideal and resistive magnetohydrodynamic stability under variable boundary conditions, Rev. Sci. Instrum. 81, 123503 (2010)

[5] O. Motojima, A. Iiyoshi, and K. Uo, Study of Current-Driven Magnetohydrodynamic Instability in the Heliotron-D Device, Phys. Rev. Lett. 44, 251 - Published 28 January 1980.

[6] Hugo Salas, Sergio Cuevas, Mariano López de Haro, Entropy generation analysis of magnetohydrodynamic induction devices, J. Phys. D: Appl. Phys. 32 (1999) 2605-2608. 